\title{
A study of the effect of wearing face masks in preventing COVID-19 transmission in the United States of America
}

\author{
Kazuyuki Suzuki \\ University of Electro-Communications, Tokyo, Japan \\ Tomonori Hasegawa \\ Toho University School of Medicine, Tokyo, Japan \\ Noriaki Kano \\ Tokyo University of Science, Tokyo, Japan, and \\ Yoshihisa Okamoto \\ Kano Quality Research Office, Tokyo, Japan
}

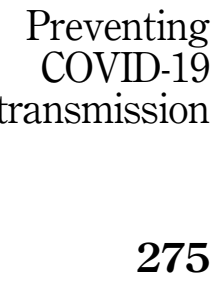

Received 26 May 2021 Revised 18 August 2021 Accepted 23 September 2021

\begin{abstract}
Purpose - The purpose of this paper is to intelligibly demonstrate the effectiveness of face mask wearing as a means to prevent COVID-19 transmission. Through understanding the benefits of wearing masks, it is hoped to facilitate the change of societal behavior and more people are willing to wear face mask.

Design/methodology/approach - The paper investigates the 50 states in the United States of America (U.S.) and Washington, D.C. that implemented the mask mandates before September 30, 2020, which are divided into four groups: (1) those implemented the statewide mask mandates before June 5, 2020 when World Health Organization (WHO) recommended mask wearing; (2) those implemented statewide mask mandates after June 5, 2020; (3) those implemented partial mandates affecting 30 percent or more of the state's population; and (4) those implemented partial mandates affecting less than 30 percent. Simple descriptive statistics are analyzed. Findings - For the 50 U.S. states and Washington, D.C., the higher the mask wearing rate, the lower the number of COVID-19 cases (correlation coefficient: -0.69 ( $p<0.001)$ ). For the 23 states with mobility reduction of less than 15 percent, the higher the proportion of population required to wear masks, the lower the number of cases. This can be seen from the difference in the number of cases among the four groups by ANOVA $(\mathrm{p}=0.013)$.

Originality - The positive effect of wearing masks is shown based on simple descriptive statistics for intuitive and intelligible understanding, which may lead people to comprehend the importance of wearing masks, and break through their custom, culture, and norms, and wear masks.
\end{abstract}

Keywords COVID-19, Mask mandate, Mask effect, Behavior change, United States

Paper type Research paper

\section{Introduction \\ COVID-19 pandemic}

During the COVID-19 pandemic, it is essential to reduce the infection risks in daily life as much as possible. COVID-19 vaccination has started in many countries, and the effect of the

(C) Kazuyuki Suzuki, Tomonori Hasegawa, Noriaki Kano and Yoshihisa Okamoto. Published in Public Administration and Policy. Published by Emerald Publishing Limited. This article is published under the Creative Commons Attribution (CC BY 4.0) license. Anyone may reproduce, distribute, translate and create derivative works of this article (for both commercial and non-commercial purposes), subject to full attribution to the original publication and authors. The full terms of this license may be seen at http:// creativecommons.org/licences/by/4.0/legalcode

This study was supported by JSPS KAKENHI Grant Number 20H02387 (Grant-in-Aid for Scientific Research (B)).
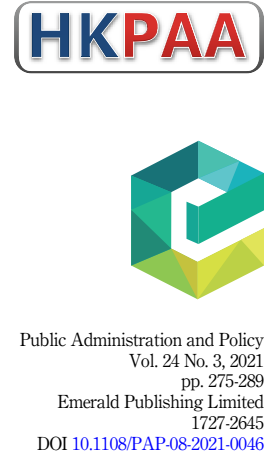
PAP

24,3

vaccines has gradually taken effect. However, even if the infection can be suppressed in some countries, eradication of COVID-19 is difficult because of increasing globalization and the appearance of coronavirus variants. It will be tough to return to a coronavirus-free society. Therefore, activities that enable society to coexist with COVID-19 are needed for the foreseeable future.

This paper will investigate the effect of face mask mandates in the U.S. on COVID-19 infection rates as a means to effect a change in societal behavior and promote the wearing of masks, which is necessary if society is to coexist with coronaviruses.

\section{Changing societal behavior}

A key concern when contemplating which measures to take against COVID-19 infection is how potential societal restrictions will affect the economy. The balance between restrictions and the economy depends on the country and the political position of its leader. For example, the former president of the U.S., Donald Trump, emphasized the economy more. In addition, it is widely known through television news and other forms of mass media that there were many Americans who did not wear masks at numerous Republican rallies before and after the presidential election. Furthermore, the implementation of such measures as mask mandates and stay-at-home orders as well as the timing of the strengthening these measures differed greatly among states depending on whether the state was under Republican or Democratic control.

\section{Purpose}

Unlike hand disinfection, social distancing and ventilation, there will be resistance to wearing face masks due to long-standing custom, social and cultural backgrounds, and subjective norms across countries or regions. In this paper, the effect of wearing masks is shown by a descriptive statistical approach so that people can easily understand and probably be convinced on the positive effect of mask wearing. Hopefully more people will break through their custom, culture, and norms and take actions of wearing masks.

\section{Research methodology}

\section{Data collection}

The effect of mask mandates on the number of COVID-19 cases was analyzed using data for the 50 U.S. states and Washington, D.C. through September 30, 2020. The data included the start date of mask mandates, the proportion of people required to wear masks, other measures taken before and after the mask mandates were implemented, the mask wearing rate and the mobility rate estimated by the Institute for Health Metrics and Evaluation (IHME, 2021), and the number of cases compiled by the Centers for Disease Control and Prevention (CDC, 2020). The authors used the IHME mask wearing rate rather than that of Carnegie Mellon University (Delphi Group, 2021) since the study focused on the period until September 30, 2020. Number of cases was standardized per million of state population (person per million / PPM). The U.S. was chosen for this study since each state makes their own health policy decisions independently, thus enabling comparison among various policies. In that sense, the U.S. can be regarded as a field for social experiments. In particular, the U.S. does not have a mask-wearing culture, and the specifics of the mask mandates are different among states.

\section{Design and statistical analysis}

First, the states and the D.C. (collectively referred to as a "state") were divided into two groups, those that implemented it throughout the state and those that implemented it in only 
certain areas (Ballotpedia, 2021; Kim et al., 2020; Markowitz, 2021; The New York Times, 2020). The former group was further divided into two groups: the states in which the mandate was implemented before June 5, 2020 when the WHO recommended mask wearing, and those in which it was implemented after June 5, 2020. Similarly, the latter group was further divided into two groups, the states with 30 percent or more of the population required to wear masks and those with less than 30 percent. In those states where the implementation date differed depending on areas, the implementation date of the county or city with the largest population was set as the start date. The population targeted for mask mandates was the total population of the areas where a mask mandate was implemented within two weeks before and after the set start date (four weeks in total). The four groups investigated are defined as follows.

Group A: Statewide mask mandate before June 5, 2021

Group B: Statewide mask mandate after June 5, 2021

Group C: 30 percent or more of the state's population required to wear a mask

Group D: Less than 30 percent of the state's population required to wear a mask

Measures such as stay-at-home orders, school closures, large-gathering prohibitions, and restaurant, bar, and recreational facility closures greatly affect mobility. Therefore, the effect of a mask mandate was examined only for the states where the mobility for four weeks (two weeks before and after the start date) was reduced by less than 15 percent compared with normal mobility. Table 1 shows the data for the 23 states with a mobility reduction rate of less than 15 percent. The number of cases two weeks after the start date was set to 100, and the increase or decrease in the number of cases was investigated for the following six weeks (from the 3rd to the 8th week). Besides, a scatter plot between the mask wearing rate for all the states and D.C. on September 30, 2020, and the number of cases for one month (September) was created, and the correlation was examined.

\section{Mask mandates start date and relevant population}

When a mask mandate was implemented in a part of the state and the implementation date of each area was different, the implementation date of the most populous area was set as the implementation date of the mask mandate in the state. When the implementation date of the mask mandate in multiple areas was consecutive, the authors compared the total population of those areas with that of the area implemented on another day before setting the state implementation date of the mask mandate. For example, in Georgia, five cities implemented a mask mandate on July 9 and two other cities did it on July 10. July 9 and 10 are consecutive and so the total population of seven cities was compared with that of the area implemented on another day. As a result, the total population of seven cities was more than the others. Next, the authors compare the population of the areas where the mask mandate was conducted on July 9 with that of the areas implemented on July 10 . Since the total population of the areas implemented on July 9 is more, July 9 was set as the implementation date of the mask mandate in Georgia. The population covered by a mandate implemented within two weeks before (including the start day) and after the start date (four weeks in total), was about 2.12 million. The total population of the state was about 10.62 million, so 20.0 percent of the population was required to wear a mask.

\section{Appearance of mask mandates effect}

Figure 1 is a time series graph showing the daily number of newly infected people per million in Ohio (Group B), South Carolina (Group C), and Nebraska (Group D). The curved line shows the seven-day moving average. In general, the effect of the mask mandate appeared about 
PAP
24,3

278

Table 1.

Portion of dataset used for analyzing effect of mask wearing (Less than $15 \%$ mobility reduction)

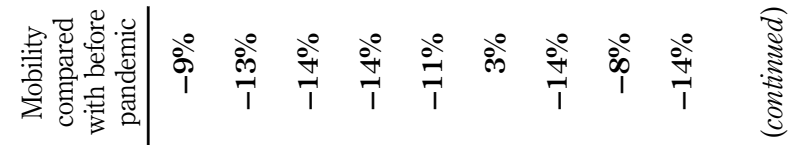

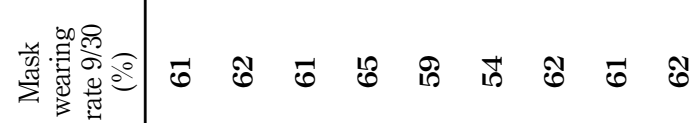

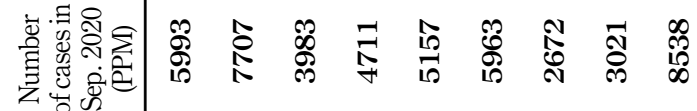

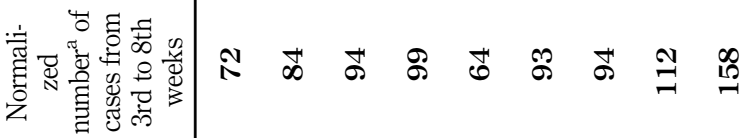

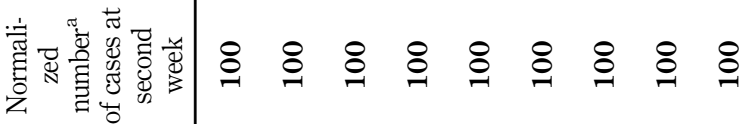

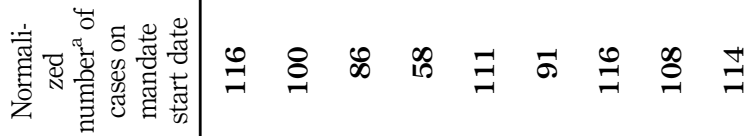

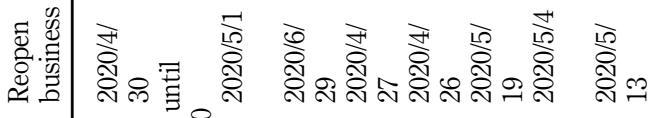

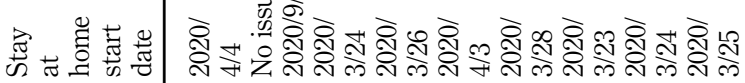

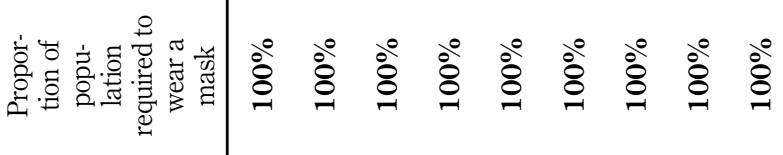

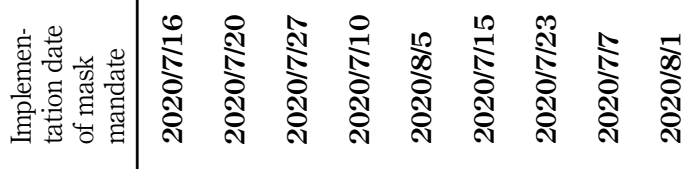

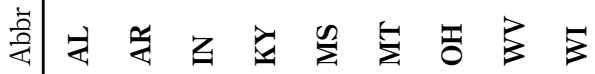

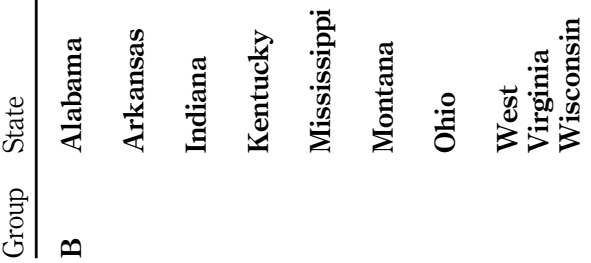




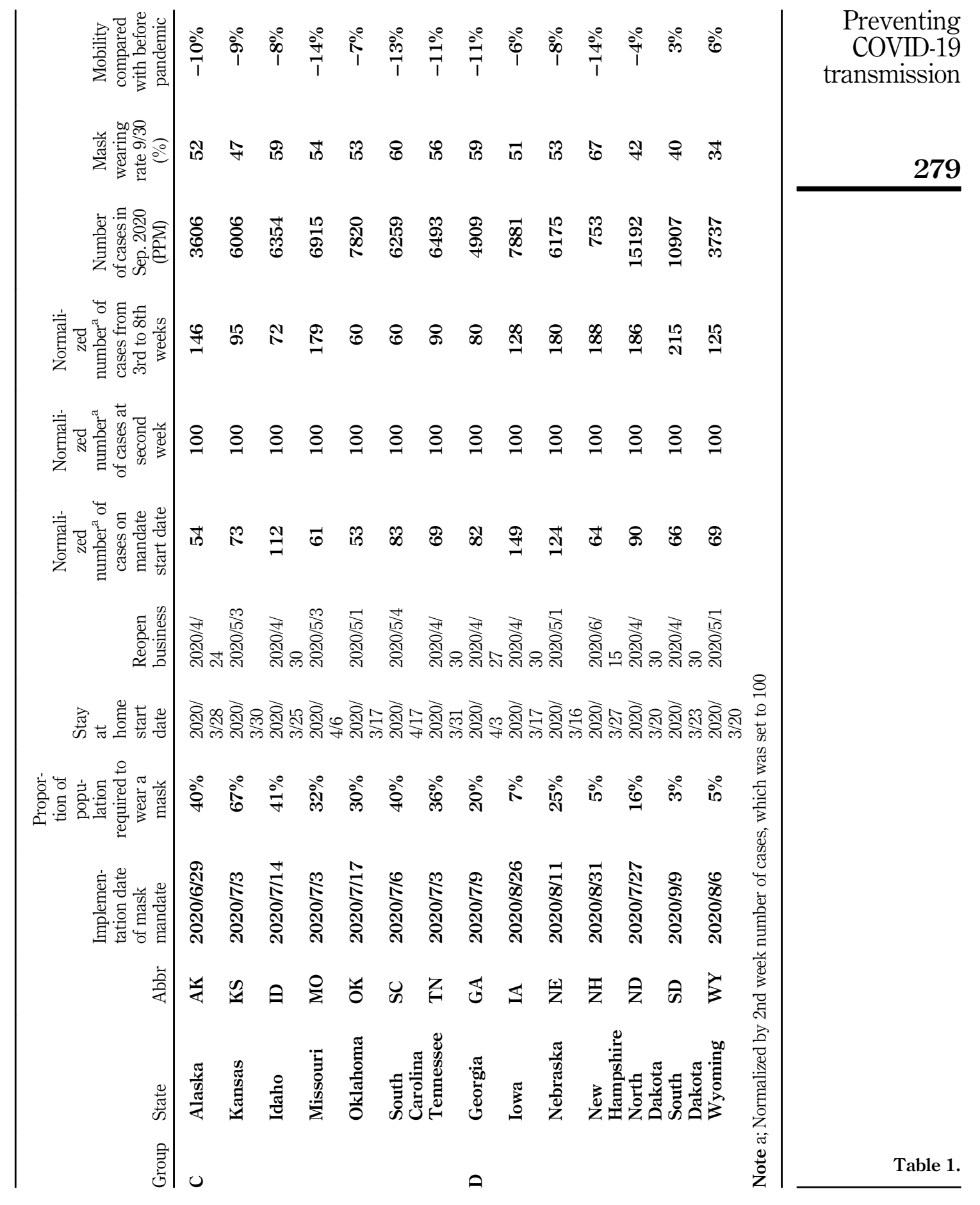


PAP

24,3

280 two weeks after the mandate start date as shown in the graph of South Carolina. That is, there was a peak in the number of cases in the second week from the start date (July 6). The same situation also occurred in Indiana, Kentucky, Montana, Kansas, Oklahoma, South Carolina, and Tennessee (Table 1). However, as shown in the table, there were several exceptions. For Alabama, Arkansas, and Idaho, the larger number of COVID-19 cases appeared on the mandate start date, which is attributed to the effect of the Independence Day holiday on July 4 , just about 2 weeks before the mandate start date. For Ohio, the larger number on the mandate start date, July 23 , is probably because seven major counties implemented mandates on July 7, about two weeks prior to statewide implementation on July 23 . The total population of the seven counties was 4.87 million of the 11.69 million state population (41.7 percent). Therefore, there was a peak in the number of cases two weeks after July 7 (Figure 1).

\section{Results}

Relationship between number of COVID-19 cases and mask mandate start date

The number of COVID-19 cases per million population in September 2020 is plotted against the mask mandate start date in Figure 2 for the four groups investigated. It is noted that each plot point in the figure represents a state or D.C. Differences in the total number of COVID-19 cases among these four groups can be clearly seen. In particular, the states that mandated mask wearing statewide before June 5 had a lower number of cases.

\section{Relationship between number of COVID-19 cases and mask wearing rate}

The number of COVID-19 cases per million people in September 2020 is plotted against the estimated mask wearing rate (IHME, 2021) on September 30, 2020 in Figure 3 for the four
Figure 1.

Time series graph of number of newly infected people in Ohio (Group B), South Carolina (Group C), and Nebraska (Group D)

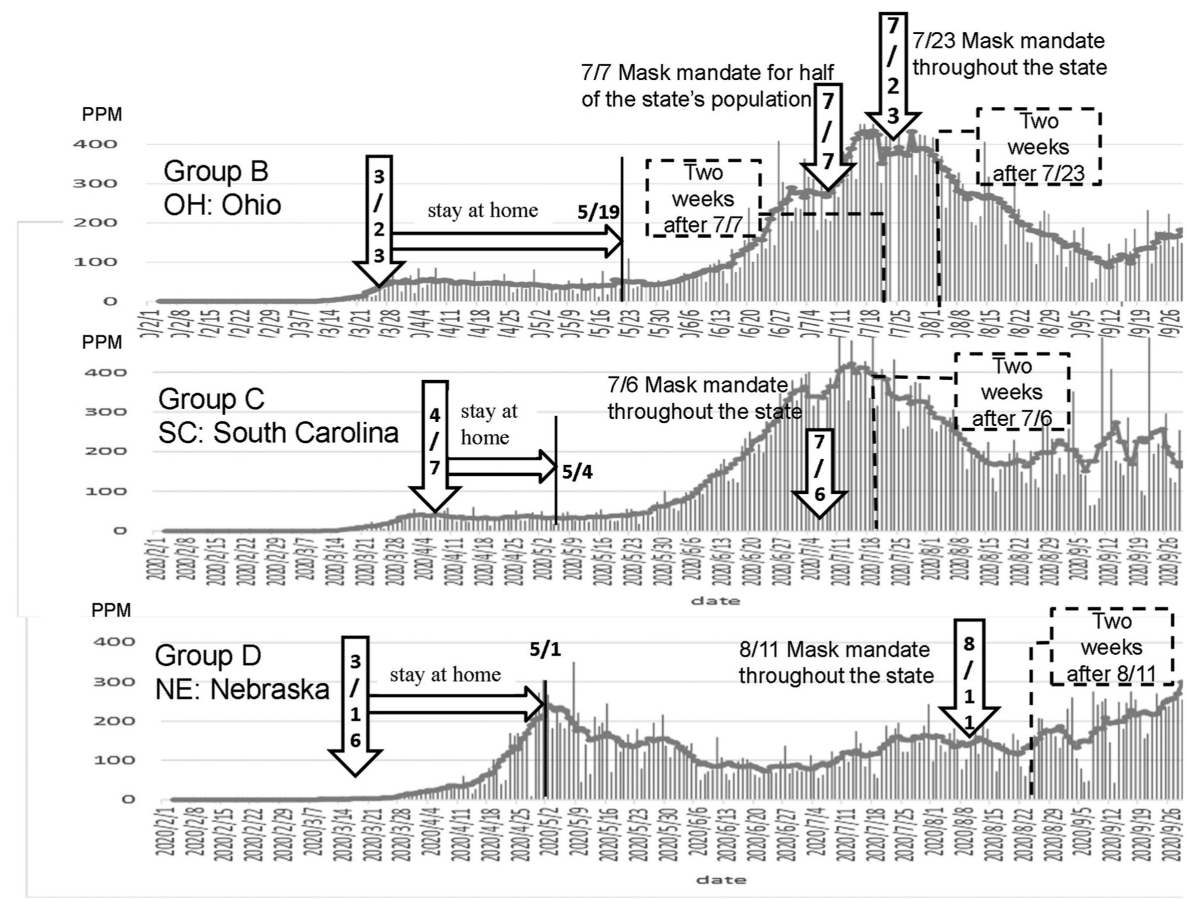




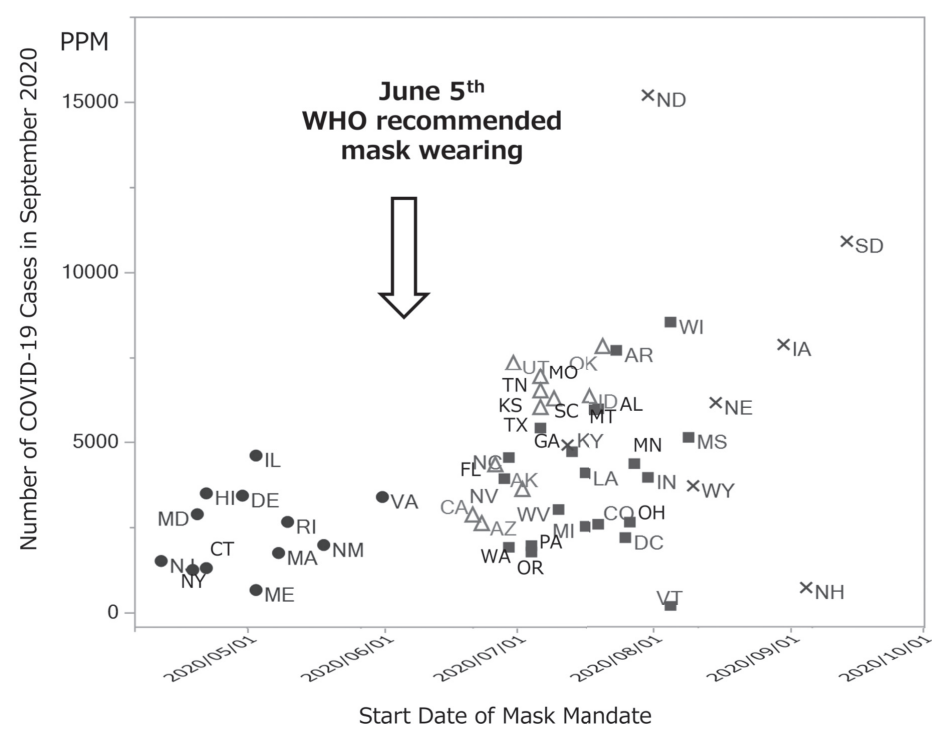

\section{Preventing \\ COVID-19 \\ transmission}

281

Group A: • Statewide, before June 5, 2020

Group B: - Statewide, after June 5, 2020

Group C: $\triangle$ Partially, $30 \%$ or more of population

Group D: $\times$ Partially, less than $30 \%$ of population

Figure 2.

Number of COVID-19 cases in September

2020 versus start date of mask mandate

groups. There is a clear correlation between the rate of mask wearing and the number of cases. The correlation coefficient for September was $-0.69(\mathrm{p}<0.001)$ including Wyoming and $-0.79(\mathrm{p}<.001)$ excluding Wyoming. That is, the higher the rate, the fewer the cases.

\section{Effect of mask mandate considering mobility}

In Figure 4, the average number of COVID-19 cases from the 3rd to the 8th weeks normalized by the 2nd week number of cases is plotted against the average mobility for four weeks (two weeks before and after the start date of mask mandates) for the 50 U.S. states and Washington, D.C. stratified by the four groups investigated. The number of cases for the 2nd week was set to 100 . The vertical dotted line corresponds to a 15 percent reduction in the mobility rate. Figure 5 shows the average mobility for four weeks (two weeks before and after the start date of mask mandates) for the 50 U.S. states and Washington, D.C. classified into the four groups. With the threshold of " -15 percent", the number of states for Groups A, B, C and D is 0, 9, 7 and 7, respectively, and they are renamed as A', B', C', and D', respectively.

Graphs for these 23 states with mobility reduction of less than 15 percent are shown in Figure 6 for the three groups described above (Groups B', C', and D', there was no state in Group A'). There was no significant difference in mobility between groups ( $p=0.109$ by ANOVA). The ANOVA result for the difference in covered population means of the groups in terms of the number of COVID-19 cases was significant $(\mathrm{p}=0.013)$, indicating that mask mandates were effective in decreasing the number of COVID-19 cases. Sensitivity analysis showed that with the threshold of the population percentage required to wear a mask changed from 30 percent to 20 percent, the ANOVA result would be still significant $(p=0.010)$. Also, if the threshold changed from 30 percent to 40 percent, the result would be still significant $(\mathrm{p}=0.045)$. 
PAP

\section{2}

Figure 3.

Number of COVID-19

cases in September 2020 versus mask wearing rate

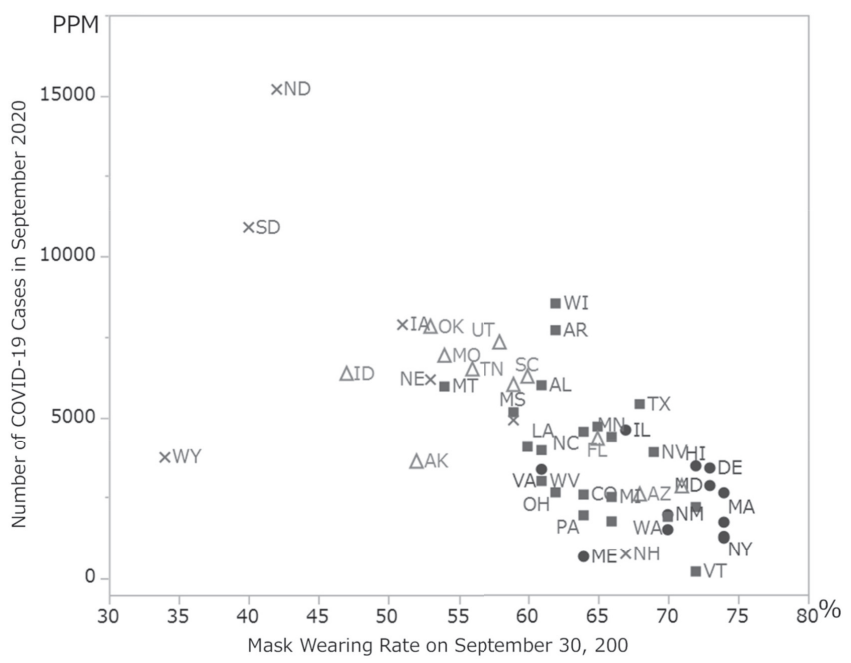

Note(s): $\mathbf{r}=-0.69(p<0.001)$ Including Wyoming $(\mathrm{WY})$ and $\mathrm{r}=-0.79$ $(p<0.001)$ Excluding Wyoming

Group A: • Statewide, before June 5, 2020

Group B: - Statewide, after June 5, 2020

Group C: $\triangle$ Partially, $30 \%$ or more of population

Group D: $\times$ Partially, less than $30 \%$ of population

\section{Discussion}

This study has three key implications as discussed below.

\section{Perceiving the benefits of mask wearing}

For changing societal behavior, Hung (2018) indicated the importance of perceived benefits of mask wearing and lack of perception of being at risk, and barriers to social interaction of mask wearing during the flu pandemic. Moreover, based on the theory of planned behavior (TPB), it was shown that risk perceptions of the pandemic and perceived benefits of face masks are the major influencing factors that positively affect public willingness to wear face masks besides attitude and social norms (Irfan et al., 2021). For perceiving the benefits of mask wearing, it is important to provide data and scientific evidence in a way that people can easily understand so that hopefully they can be convinced of the genuine need to wear a mask.

Face coverings can have both social and cultural meanings. In the U.S, mask mandates have become a political issue because being required to wear a mask in public is thought by many people to be a violation of freedom (Rojas, 2020). Wearing one has been described as dehumanizing as well (MacFarquhar, 2020). Several U.S. government officials including the former president, Donald Trump, initially refused to wear a mask, describing it as a sign of weakness. They asserted their authority by not wearing a mask (Ball, 2020).

Social and community thinking may affect mask wearing. For example, the mask wearing rate varied among states depending on whether the state supported the Republican or Democratic party in the 2020 U.S. presidential election. Figure 7 shows the same data as 


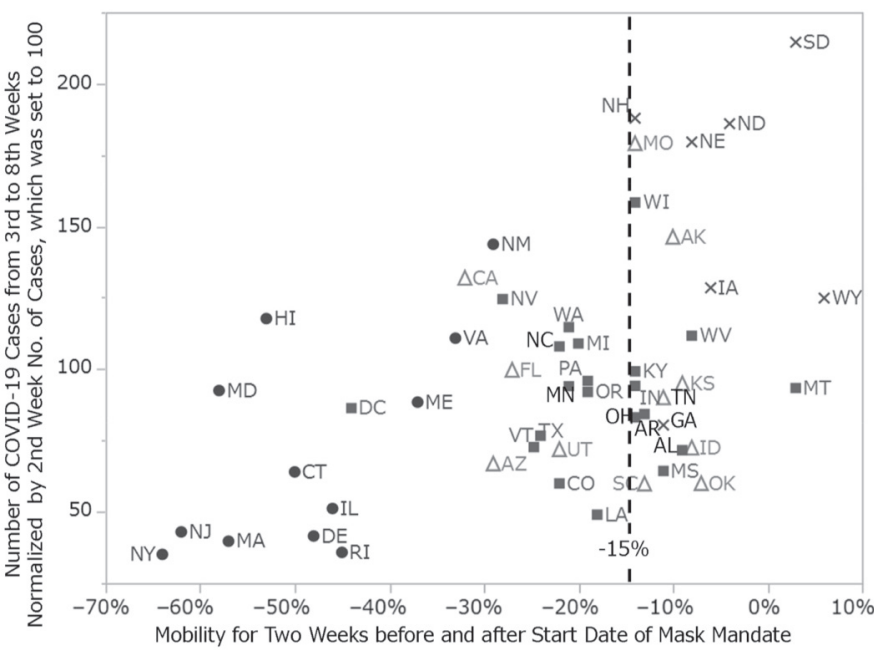

Group A: • Statewide, before June 5, 2020

Group B: - Statewide, after June 5, 2020

Group C: $\triangle$ Partially, $30 \%$ or more of population

Group D: $\times$ Partially, less than $30 \%$ of population

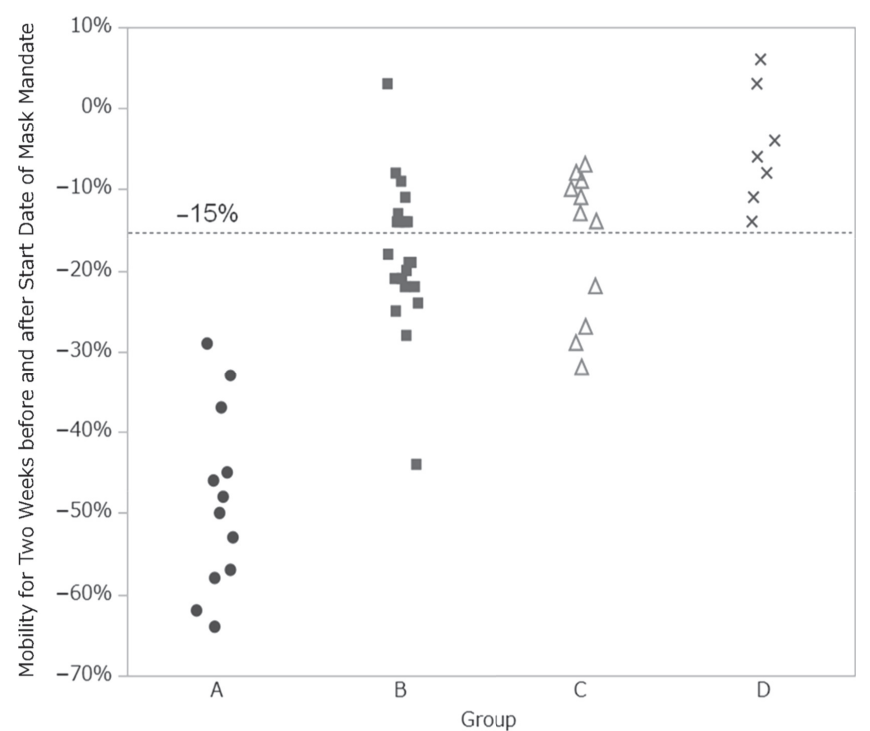

Group A: • Statewide, before June 5, 2020

Group B: - Statewide, after June 5, 2020

Group C: $\triangle$ Partially, $30 \%$ or more of population

Group D: $\times$ Partially, less than $30 \%$ of population
Preventing

COVID-19

transmission

283

Figure 4.

Number of COVID-19

cases from 3rd to 8th weeks versus mobility
Figure 5.

Average mobility for four weeks (two weeks before and after the start date of mask mandates) for the 50 U.S. states and Washington, D.C. stratified by the four groups 
PAP

24,3

\section{4}

Figure 6.

Comparison of normalized number of COVID-19 cases by different population groups required to wear a mask

Figure 7.

Number of COVID-19 cases in September 2020 versus mask wearing rate stratified by political party supported in 2020 U.S. presidential election

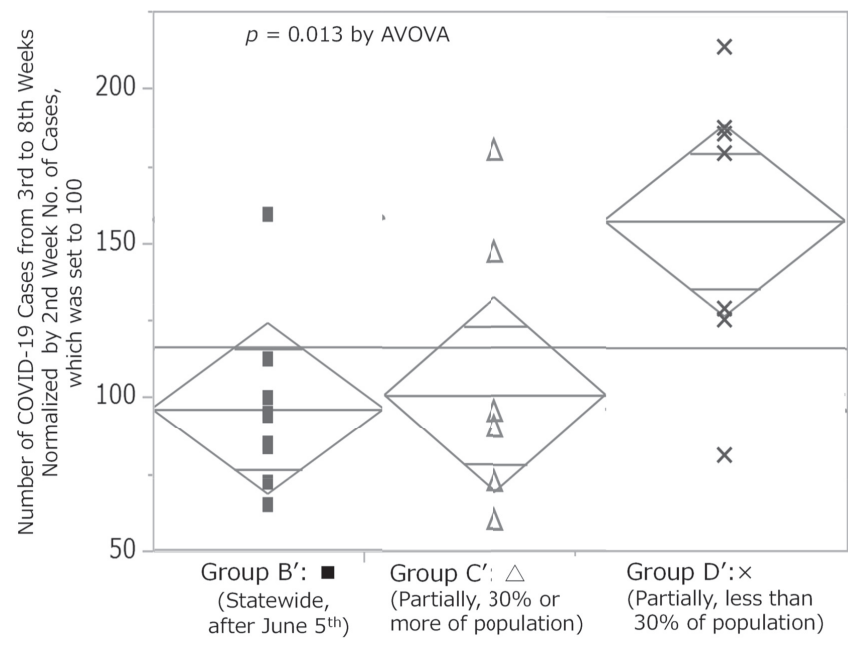

Note(s): Mobility was limited to less than $15 \%$; Graphs show point estimates and $95 \%$ confidence intervals of population means

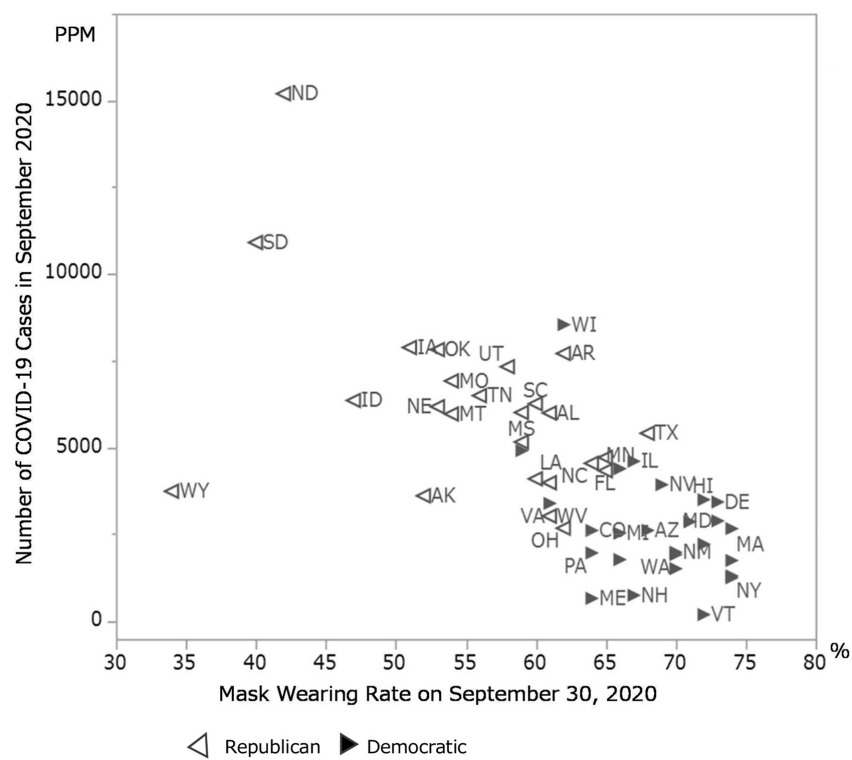

Figure 3, with the states labeled with the party they supported in the 2020 presidential election. The states in which self-responsibility is highly valued were likely to have supported the Republican Party and that valuation may have influenced the way the residents think about wearing a mask. Schoeni et al. (2021) showed that both the rate of not wearing a mask in the past week and the adjusted percentage of at-risk adults never wearing a mask were higher for Republicans than Democrats. Also, Green et al. (2020) showed that Democratic members of 
congress were more likely to frame the pandemic as a public health threat than Republican members.

As shown in this study, protecting public health requires compromising individual freedom although individual freedom should not be taken lightly. This is because people who do not wear a mask present a higher risk of infection to others. This is analogous to smokers presenting a risk to non-smokers through passive smoking. The similarities between mask wearing and smoking are discussed in the literature (Vuolo et al., 2020). Bavel et al. (2020) also focused on protecting others, besides several social scientific insights such as emphasizing benefits to the recipient, aligning with the recipient's moral values and appealing to social consensus or scientific norms. To bring some advantages to other people, using evolutionary game theory, Kabir et al. (2021) quantified how people use mask-wearing and related protecting behaviors. To promote mask wearing for protecting others, it is important to provide data and scientific evidence in an easy-to-understand way. Behavior change is a multi-step, multi-factor process, and how to incorporate scientific findings into public health policy is an issue for future study.

\section{Differences from previous research}

Previous studies on the effects of mask wearing were summarized by Brooks and Butler (2021) from an epidemic point of view. Lyu and Wehby (2020) focused on requirements to wear a mask in public as well as restrictions on movement such as banning gatherings and closing schools. Using a model based on an exponential growth curve, they found that the increase in the rate of COVID-19 infections was possibly reduced by up to 2.0 percent by implementing mask mandates. X. Wang et al. (2020) and Y. Wang et al. (2020) used a weighted nonlinear regression model, and a multivariable logistic regression model respectively, to show the effect of mask wearing. Model-based analysis and a descriptive statistical approach used in this paper have a complementary relationship. In formulating health policy, it is important to make decisions based on the knowledge obtained by various methods in view of the magnitude of the impact.

To investigate the effects of mask wearing, Lyu and Wehby (2020) focused on the number of days since the declaration of a mask mandate while Gallaway et al. (2020) focused on the number of days since the start date of a mandate. This study used the latter approach in order to reveal the effects of mask mandates more clearly. While the study of Gallaway et al. (2020) was limited to one U.S. state, this study expanded the scope to all U.S. states plus D.C.

\section{Effectiveness of early mask mandates}

As can be seen from Figure 2, the earlier the mask mandates were issued by the states, the smaller the number of infected people. Also, from Figure 3, it is found that the higher the mask wearing rate, the smaller the number of infected people. The above result may be attributed to whether the state supported the Republican or Democratic Party in the 2020 U.S. presidential election from Figure 7. On the other hand, the graphs of mask wearing rates on the IHME web site (IHME, 2021) revealed that once statewide mask mandates were implemented, the mask wearing rate either increased or stayed the same for the most part. The mask wearing rates varied depending on the state, however, it rarely decreased before the end of September. This further demonstrates how important mask mandate was in changing people's behavior within a certain period.

\section{Mechanism of infection prevention by masks}

The transmission of COVID-19 can be roughly divided into three main routes: droplet infection by release of droplets $5 \mu$ or more in diameter from the oral cavity, aerosol infection
Preventing

COVID-19

transmission 
PAP

24,3

286

caused by droplet nuclei (less than $5 \mu$ in diameter) formed due to evaporation of the moisture in droplets due to low humidity (particularly in winter), and contact infection due to touching the face and eyes. For droplet infection, masks and social distancing are effective. For aerosol infection, ventilation and safety glasses in addition to masks are effective. For contact infection, hand hygiene is definitely important. Also, masks are effective since wearing a mask helps to reduce the frequency of touching the face (Chen et al., 2020). In short, masks are indispensable items for all three infection routes.

In addition, from the micro perspective, the effectiveness of mask wearing should be considered on the basis of various factors such as the mask design and material, the manufacturing process, humidity, ventilation, and population density. These factors are addressed in the research literature (Riken, 2020; Ueki et al., 2020). Various experiments as well as a descriptive statistical approach are indispensable to facilitate people's understanding on the effects of mask wearing.

\section{Strengths and limitations}

There are two strengths of this study. First, the analysis method is based on descriptive statistical approach to demonstrate the effect of mask wearing using comprehensively summarized data in each U.S. state and D.C. This study makes it easier for people to understand the importance of mask wearing. Hopefully, this could contribute to probable changes in mask wearing behavior.

Second, regarding the advantage of wearing masks, an important point is that masks do not substantially interfere with economic activities if appropriate measures are taken related to eating, drinking, singing, speaking, yelling, etc. Since infection spreads through human movement and face-to-face communication, infection can be prevented by using virtual communication like web conferencing under stay-at-home orders that restrict the movement of people (Kano et al., 2020)). However, real face-to-face communication is difficult. In addition, under various restrictions, the economic activities of travel-related businesses including railways, airlines, and hotels are limited. Masks are an effective way to reduce these difficulties.

This study is an ecological study and thus does not directly explain causal relationships. Moreover, factors other than mask wearing may have affected the results. The two main limitations are as follows. First, there are effects other than masks. In addition to promoting mask wearing and limiting mobility, it is important to enforce social distancing to prevent droplet infection, improve ventilation to prevent aerosol infection, and disinfect surfaces to prevent contact infection. This study set a four-week mobility condition (two weeks before and after the start date of mask mandates), i.e., a reduction in mobility of less than 15 percent, and made no assumptions about other major changes in behavior such as disinfecting surfaces, improving ventilation, and social distancing. In addition, the effect of being vaccinated on mask wearing was not considered.

Second limitation is related to mask wearing rate, how to wear, and the types of mask. According to the data on mask wearing from IHME (2021), on September 30, 2020, the maximum rate of mask wearing was 77 percent in the U.S., much less than 100 percent. Although infection rates are affected by the types of mask worn and the way in which masks are worn (Tamamoto et al., 2020), measures aimed at improving the mask wearing rate, using effective masks, and wearing masks properly were not taken into account.

\section{Conclusion}

The effectiveness of mask wearing was investigated using data for the 50 U.S. states and Washington, D.C. through September 30, 2020. The effect of mask mandate was clarified 
under a similar mobility condition, thereby excluding the effects of restrictions on movement such as stay-at-home orders and gathering limitations. This study revealed that the higher the mask wearing rate, the lower the number of COVID-19 cases, and the higher the proportion of population required to wear masks, the lower the number of cases. Mask wearing could last for a relatively long period of time once a mask mandate is declared. Presenting the effects in an easy-to-understand manner may lead to better understanding by the general public of the effectiveness of mask wearing and to changes in their mask-wearing behavior. This approach may prove useful in promoting measures aimed at controlling other types of infections as well.

\section{References}

Ball, P. (2020), "How mask-wearing became a new culture war", New Statesman, 15 July, available at: https://www.newstatesman.com/politics/uk/2020/07/how-mask-wearing-became-new-culturewar (accessed 29 April 2021).

Ballotpedia (2021), "State-level mask requirements in response to the coronavirus (COVID-19) pandemic, 2020-2021", available at: https://ballotpedia.org/State-level_mask_requirements_in response_to_the_coronavirus_(COVID-19)_pandemic,_2020-2021 (accessed 29 April 2021).

Bavel, J.J.V., Baicker, K., Boggio, P.S., et al. (2020), "Using social and behavioural science to support COVID-19 pandemic response", Nature Human Behaviour, Vol. 4, pp. 460-471.

Brooks, J.T. and Butler, J.C. (2021), "Effectiveness of mask wearing to control community spread of SARS-Cov-2", The Journal of the American Medical Association, Vol. 325 No. 10, pp. 998-999.

Centers for Disease Control and Prevention (CDC) (2020), "United States COVID-19 cases and deaths by state over time", available at: https://data.cdc.gov/Case-Surveillance/United-States-COVID19-Cases-and-Deaths-by-State-o/9mfq-cb36/ (accessed 29 April 2021).

Chen, Y.J., Qin, G., Chen, J., et al. (2020), "Comparison of face-touching behaviors before and during the coronavirus disease 2019 pandemic", JAMA Network Open, Vol. 20 No. 7, pp. 1-10, doi: 10.1001/ jamanetworkopen.2020.16924.

Delphi Group, Carnegie Mellon University (2021), "Real-time indicators of COVID-19 activity", available at: https://delphi.cmu.edu/ (accessed 29 April 2021).

Gallaway, M.S., Rigler, J., Robinson, S., et al. (2020), "Trends in COVID-19 incidence after implementation of mitigation measures - Arizona, January 22-August 7, 2020”, The Morbidity and Mortality Weekly Report, Vol. 69 No. 40, pp. 1460-1463.

Green, J., Edgerton, J., Naftel, D., Shoub, K., and Cranmer, S.J. (2020), "Elusive consensus: polarization in elite communication on the COVID-19 pandemic", Science Advances, Vol. 6 No. 28, pp. 1-5, doi: 10.1126/sciadv.abc2717.

Hung, Y. (2018), "A study of barriers to the wearing of face masks by adults in the US to prevent the spread of influenza", Unpublished Master thesis, Arizona State University, available at: https:// repository.asu.edu/attachments/211332/content/Hung_asu_0010N_18395.pdf (accessed 29 April 2021).

Institute for Health Metrics and Evaluation (IHME) (2021), "COVID-19 projections", available at: https://covid19.healthdata.org/ (accessed 29 April 2021).

Irfan, M., Akhtar, N., Ahmad, M., Shahzad, F., Elavarasan, R.M., Wu, H. and Yang, C. (2021), "Assessing public willingness to wear face masks during the COVID-19 pandemic: fresh insights from the Theory of Planned Behavior", International Journal of Environmental Research and Public Health, Vol. 18 No. 9, pp. 1-22, doi: 10.3390/ijerph18094577.

Kabir, K.M.A., Risa, T. and Tanimoto, J. (2021), "Prosocial behavior of wearing a mask during an epidemic: an evolutionary explanation", Scientific Reports, Vol. 11, pp. 1-14, doi: 10.1038/s41598021-92094-2.

\section{Preventing \\ COVID-19 \\ transmission}


PAP

24,3

288

Kano, N., Suzuki, K., Hasegawa, T. and Okamoto, Y. (2020), "Building society quality towards withCovid-19 society", keynote paper presented at ANQ Congress 2020, 22 October, Seoul, available at: https://anforq.org/media/Plenary-Session-I_Presentation_from_Dr_Noriaki_Kano.pdf (accessed 20 April 2021).

Kim, A., Andrew, S. and Froio, J. (2020), "These are the states requiring people to wear masks when out in public", available at: https://edition.cnn.com/2020/06/19/us/states-face-mask-coronavirustrnd/index.html (accessed 29 April 2021).

Lyu, W. and Wehby, G.L. (2020), "Community use of face masks and COVID-19: evidence from a natural experiment of State Mandates in the US", Health Affairs (Millwood), Vol. 39 No. 8, pp. 1419-1425.

MacFarquhar, N. (2020), "Who's enforcing mask rules? Often retail workers, and they are getting hurt", The New York Times, 15 May, available at: https://www.nytimes.com/2020/05/15/us/ coronavirus-masks-violence.html?auth=login-email\&login=email (accessed 29 April 2021).

Markowitz, A. (2021), "State-by-State Guide to face mask requirements, American Association of Retired Persons", available at: https://www.aarp.org/health/healthy-living/info-2020/statesmask-mandates-coronavirus.html (accessed 29 April 2021).

Riken Center for Computational Science (2020), “COVID-19: wear mask \& ventilate' said Fugaku”, available at: https://www.r-ccs.riken.jp/en/fugaku/research/covid-19/msg-en/ (accessed 29 April 2021).

Rojas, R. (2020), "Masks become a flash point in the virus culture wars", The New York Times, 3 May, available at: https://www.nytimes.com/2020/05/03/us/coronavirus-masks-protests.html (accessed 29 April 2021).

Schoeni, R.F., Wiemers, E.E., Seltzer, J.A. and Langa, K.M. (2021), "Political affiliation and risk taking behaviors among adults with elevated chance of severe complications from COVID-19", Preventive Medicine, Vol. 153, pp. 1-5, doi: 10.1016/j.ypmed.2021.106726.

Tamamoto, K.A., Rousslang, N.D., Ahn, H.J., Better, H.E. and Hong, R.A. (2020), "Public compliance with face mask use in Honolulu and regional variation”, The Hawai i Journal of Health \& Social Welfare, Vol. 79 No. 9, pp. 268-271.

The New York Times (2020), "See coronavirus restrictions and mask mandates for all 50 states", 25 April.

Ueki, H., Furusawa, Y., Iwatsuki-Horimoto, K., et al. (2020), "Effectiveness of face masks in preventing airborne transmission of SARS-CoV-2", mSphere, Vol. 5 No. 4, pp. 1-5, doi: 10.1128/mSphere. 00637-20.

Vuolo, M., Kelly, B.C. and Roscigno, V.J. (2020), "COVID-19 mask requirements as a workers' rights issue: parallels to smoking bans", America Journal of Preventive Medicine, Vol. 59 No. 5, pp. 764-767.

Wang, X., Ferro, E.G., Zhou, G., Hashimoto, D. and Bhatt, D.L. (2020), "Association between universal masking in a health care system and SARS-CoV-2 positivity among health care workers", The Journal of the American Medical Association, Vol. 324 No. 7, pp. 703-704, doi: 10.1001/jama. 2020.12897.

Wang, Y., Tian, H., Zhang, L., et al. (2020), "Reduction of secondary transmission of SARS-CoV-2 in households by face mask use, disinfection and social distancing: a cohort study in Beijing, China”, BMJ Glob Health, Vol. 5, pp. 1-9, doi: 10.1136/bmjgh-2020-002794.

\footnotetext{
About the authors

Kazuyuki Suzuki, PhD, is Professor Emeritus at the University of Electro-Communications, Tokyo. His academic expertise covers reliability engineering and quality management. He has served as both the President of the Japanese Society for Quality Control (2010, 2011), and the President of Reliability Engineering Association of Japan (2012, 2013). He published more than 80 reviewed articles and 35 books besides 31 keynote speeches and plenary invited talks. He won 18 prizes from several academic societies including the Deming Prize for Individual in 2014, and Wilcoxon Prize in 1999 from the
} 
American Society for Quality and American Statistical Association. Kazuyuki Suzuki is the corresponding author and can be contacted at: suzuki@uec.ac.jp

Tomonori Hasegawa, $\mathrm{MD}, \mathrm{PhD}$, is Professor of the Department of Social Medicine, Toho University, School of Medicine. His academic expertise covers health policies and performance evaluation of health systems. He published about 200 reviewed articles and about 100 books. He was engaged in health sector reform in Japan as an advisory member of the Cabinet Office (2001-2010). He is an executive board member of the Japan Council for Quality Health Care, a board member of the Japanese Society of Healthcare Management, Editor-in-Chief of the Journal of Japanese Healthcare Management.

Noriaki Kano, PhD, is Professor Emeritus of Tokyo University of Science. His outstanding achievement was his advocacy of attractive quality theory (Kano Model). His expertise covers Quality Management/Engineering. He has been Honorary Chairperson of Asian Network for Quality (ANQ) and nominated as Honorary Positions from 17 quality organizations including International Academy for Quality, American Society for Quality, and China Association for Quality. He authored over 300 publications. He was awarded 9 international awards including the 4 annually highest quality awards in each of America, Europe, Japan and Asian-Pacific. He has three eponymous awards including IshikawaKano Award established by ANQ.

Yoshihisa Okamoto, M.Eng., is guest expert at the Kano Quality Research Office. His academic expertise covers quality management. He is engaging in quality assurance for over 15 years in automotive industry. He is an academic member of Japanese Society for Quality Control.

For instructions on how to order reprints of this article, please visit our website: 